\title{
Advances in Arbovirus Surveillance, Detection and Diagnosis
}

\author{
Roy A. Hall, ${ }^{1}$ Bradley J. Blitvich, ${ }^{2}$ Cheryl A. Johansen, ${ }^{3}$ and Stuart D. Blacksell ${ }^{4}$ \\ ${ }^{1}$ Australian Infectious Diseases Research Centre, School of Chemistry and Molecular Biosciences, The University of Queensland, \\ St Lucia. QLD 4072, Australia \\ ${ }^{2}$ Department of Veterinary Microbiology and Preventive Medicine, Iowa State University, 2116 Veterinary Medicine, Ames, \\ IA 50011-1250, USA \\ ${ }^{3}$ Arbovirus Surveillance and Research Laboratory, Discipline of Microbiology and Immunology M502, School of Biomedical, \\ Biomolecular and Chemical Sciences, The University of Western Australia, Nedlands, WA 6009, Australia \\ ${ }^{4}$ Mahidol-Oxford Tropical Medicine Research Unit (MORU), Faculty of Tropical Medicine, Mahidol University, 420/6 Rajavithi Road, \\ Rajathawee District, Bangkok 10400, Thailand
}

Correspondence should be addressed to Roy A. Hall, roy.hall@uq.edu.au

Received 12 February 2012; Accepted 12 February 2012

Copyright (C) 2012 Roy A. Hall et al. This is an open access article distributed under the Creative Commons Attribution License, which permits unrestricted use, distribution, and reproduction in any medium, provided the original work is properly cited.

Arthropod-borne viruses (arboviruses) are responsible for many important vector-borne diseases of man and animals including dengue, yellow fever, Japanese encephalitis, tickborne encephalitis, Rift Valley fever, West Nile fever, chikungunya, Ross River disease, and bluetongue. Detection or accurate prediction of virus activity in vector populations and specific diagnosis of infection in the human or animal host are crucial components of effective control and treatment strategies and facilitate early warning of potential or existing outbreaks and initiation of vector management and/ or vaccination programs.

One of the key elements of the control of arbovirus transmission is early detection of virus activity or increased virus activity in vector populations. Surveillance programs designed to monitor these parameters provide an early warning system of increased risk of transmission and disease outbreak. One approach routinely used by groups to monitor virus activity is sentinel animal surveillance. This is particularly useful for veterinary or zoonotic arboviruses where domestic animals (e.g., cattle, pigs or chickens) can be effectively employed. While serological monitoring of strategically positioned flocks or herds can provide early warning of virus transmission in a specific region, there are many difficulties with this system: the costs of maintaining the animals (particularly in remote areas), lack of specificity of serological assays, and the ability to only target viruses that infect the selected sentinel animal or those that are transmitted by vectors that feed on the sentinel host. To address these problems, a new approach (reviewed by A. F. van den Hurk and colleagues in this issue) has recently been applied to the specific detection of virus activity in mosquito populations, which is particularly useful for remote locations. The specific detection of viral RNA expectorated by infected mosquitoes feeding on a sugar-coated matrix has enabled these investigators to collect samples over prolonged periods in remote locations and specifically identify viruses carried by any mosquito feeding on the sugar bait. The potential application of this system to a wide variety of arbovirus surveillance scenarios is very promising.

Another important component of arbovirus surveillance is the detection of virus in arthropod vector populations. Traditional methods include trapping vectors such as mosquitoes, ticks, and midges, identifying them to species level and analysing vector pools for known viruses of interest. Virus isolation by inoculating mice, embryonated eggs, or colonized vector species has largely been replaced by in vitro methods (e.g., inoculation of arthropod and vertebrate cell lines), but is still the most effective means for monitoring virus activity for some viruses. However, the enhanced technologies for the specific detection of viral nucleic acid, such as multiplexed real-time PCR protocols, provide more rapid, sensitive, and specific approaches for detecting virus activity in vector populations. Furthermore, the recent application of next-generation sequencing technologies to rapidly analyze 
nucleic acid of unidentified viral isolates provides a revolutionary approach for the discovery and genetic characterization of new vector-borne viruses.

Monitoring arthropod vector populations is also an important component of arbovirus surveillance, particularly for detecting an increase in known vectors or the introduction of a new species into an area (e.g., recent incursions of Aedes albopictus into Europe and Australia). In addition to the labor-intensive methods of trapping and identifying vectors by morphology or genetic analysis, new strategies such as satellite-based remote sensing of vector breeding sites and assessment of the risk of virus transmission based on proximity to human or animal habitation (see the paper by Susan $\mathrm{N}$. Rossmann and colleagues in this issue) can rapidly provide highly useful data on a very large scale.

Accurate and timely diagnosis of arbovirus infections is also crucial to ensure appropriate patient management, for the reporting of virus activity in a region and to allow instigation of control strategies such as vector management, vaccination, and public awareness campaigns. Serological assays are predominantly used for this purpose; however, many problems exist with traditional methods including the use of live virus for antigen production and plaque reduction neutralization tests, lack of specificity due to crossreactivity between related viruses, and the costs of running individual assays for each viral antigen used. However the use of recombinant viral antigens (whole proteins, domains or peptides) in multiplexed formats such as microsphere immunoassays provides rapid, sensitive, and specific analyses that can be coupled to large-scale antigen production methods (see the paper by J. He and colleagues in this issue) and high-throughput robotic systems in the diagnostic laboratory. For some viral infections, enhanced real-time PCR protocols provide a more specific alternative with the detection of viral nucleic acid in human or animal samples (see the review by N. Johnson and colleagues in this issue). The availability of several commercial point-of-care assays, particularly for dengue and West Nile viruses, also provides a useful tool for the clinician or veterinarian treating the patient on presentation at the clinic (see the reviews by S. D. Blacksell and J. M. Hobson-Peters in this issue).

The new technologies and novel approaches referred to above and elaborated on in the papers in this special issue provide an excellent platform for the advancement of arbovirus surveillance and diagnosis. Thorough evaluation of their effectiveness against traditional methods in the field and clinic and their application to different arboviral diseases will allow their routine implementation and unleash the potential to vastly improve our ability to manage these diseases in the future.

Roy A. Hall

Bradley J. Blitvich

Cheryl A. Johansen

Stuart D. Blacksell 

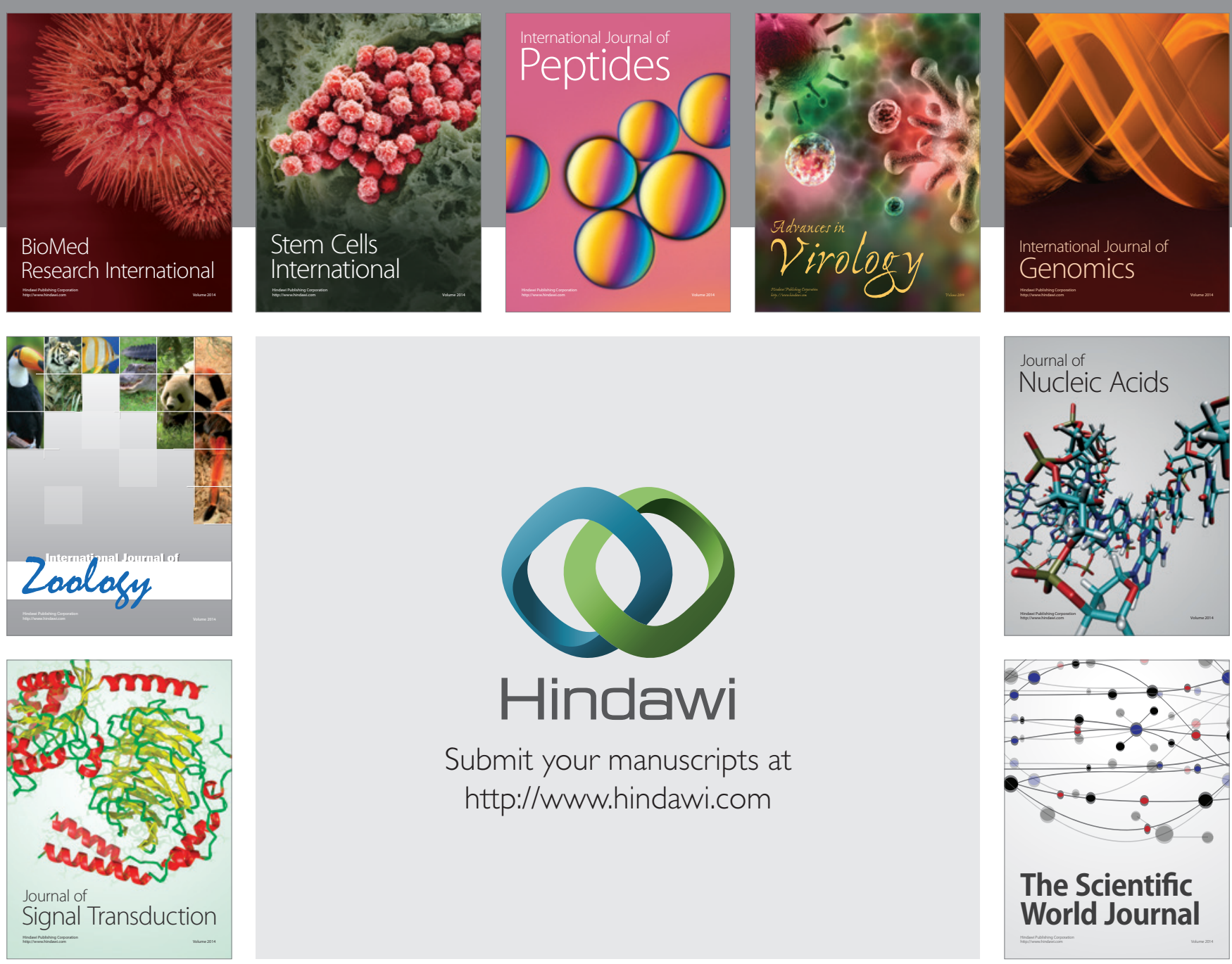

Submit your manuscripts at

http://www.hindawi.com
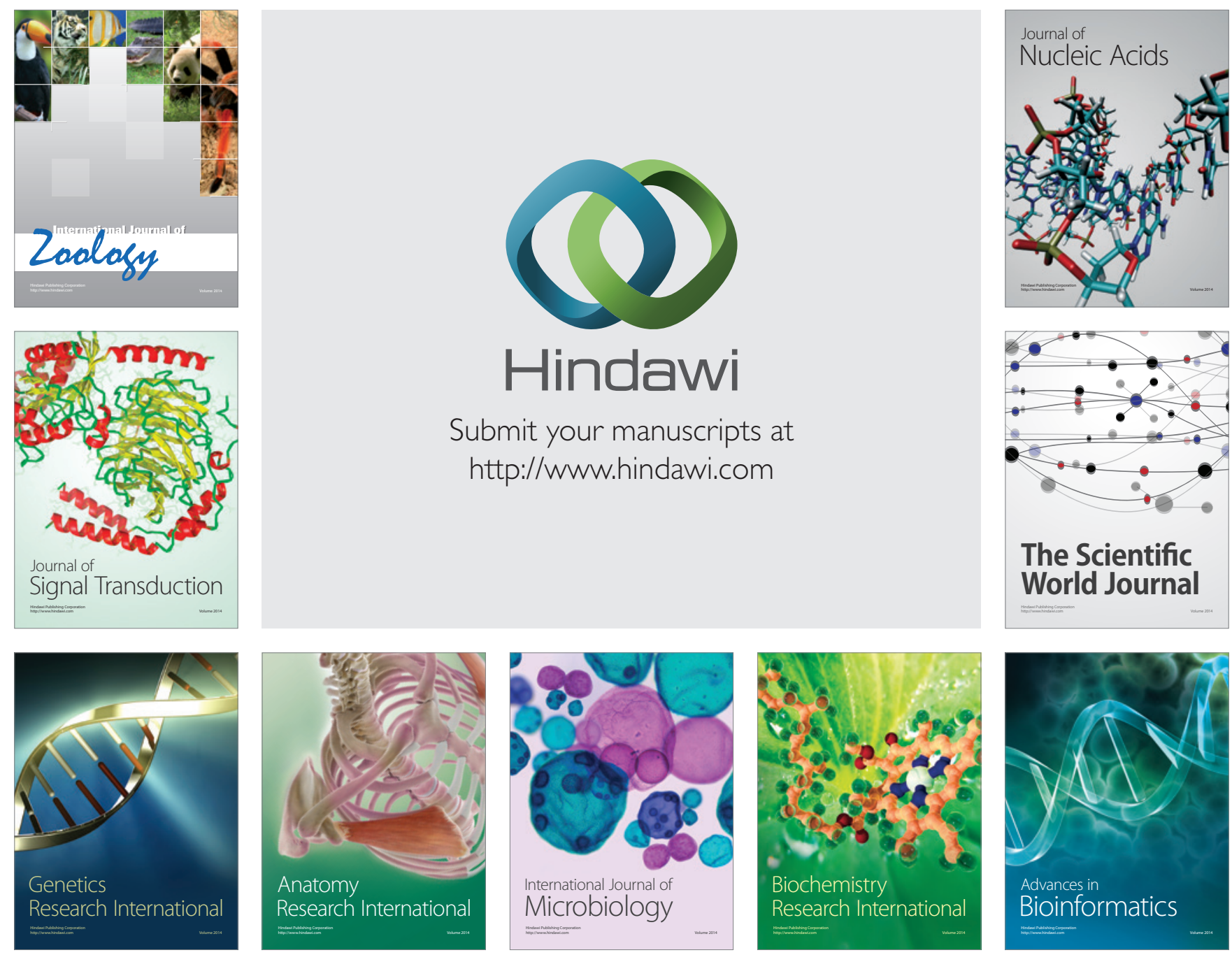

The Scientific World Journal
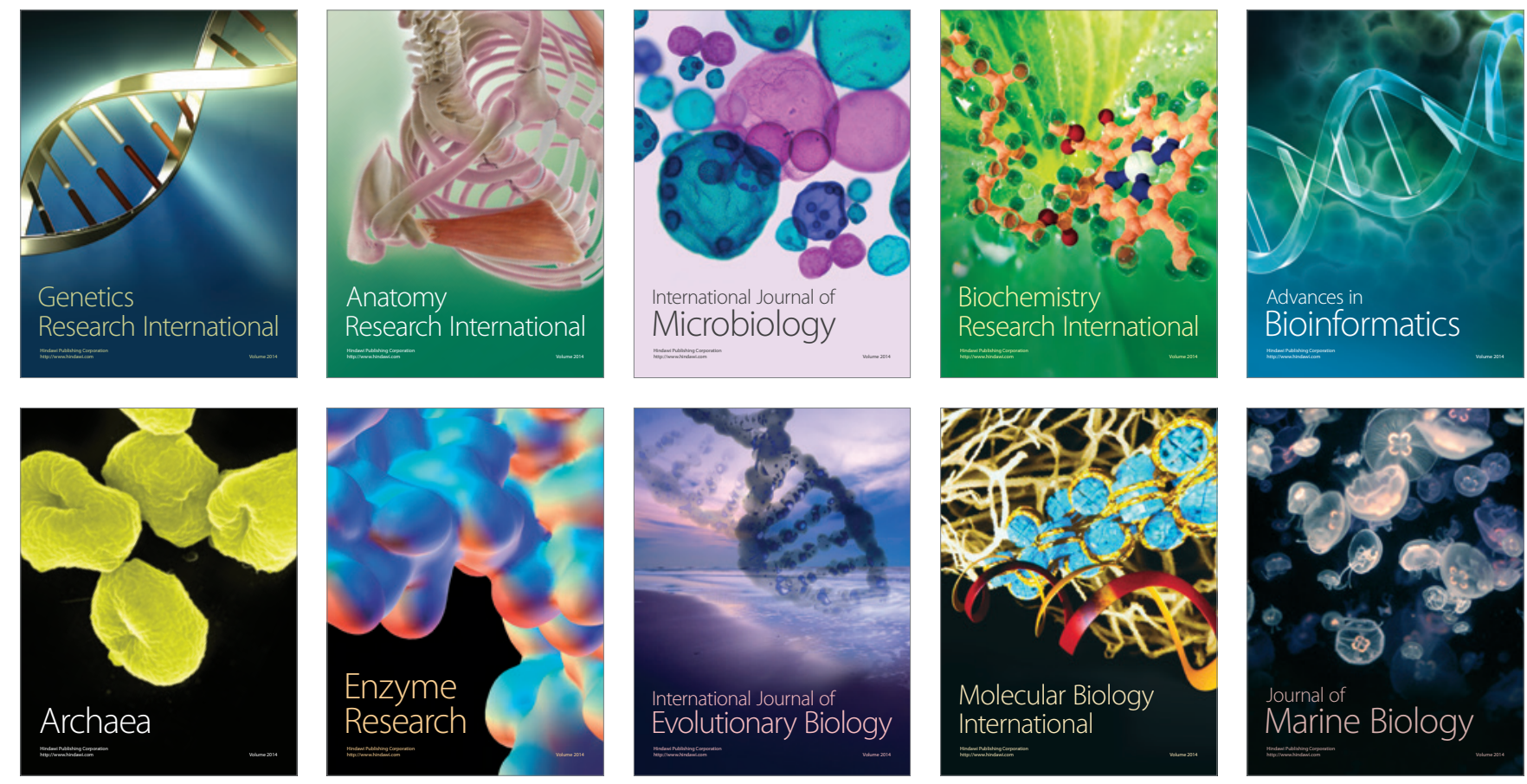